\title{
Clarification to provide further understanding of the conduct and design of TROPIC: A Phase 3 trial of cabazitaxel versus mitoxantrone in patients with metastatic castration-resistant prostate cancer
}

Dear Editors,

We read with interest the article "Efficacy and safety of second-line agents for treatment of metastatic castration-resistant prostate cancer progressing after docetaxel. A systematic review and meta-analysis" by Perletti et al. in your journal (Arch Ital Urol Androl. 2015; 87:121-9), which describes the results of a systematic literature review assessing second-line agents used in the treatment of metastatic castration-resistant prostate cancer (mCRPC) progressing after docetaxel-based regimens. The authors analyzed ten articles reporting the results of five Phase 3, randomized, controlled trials that enrolled patients with mCRPC progressing during or after first-line docetaxel treatment. This included the Phase 3 TROPIC trial of cabazitaxel versus mitoxantrone (1), which provided the basis for the regulatory approval of cabazitaxel in this setting.

The authors conducted a risk of bias analysis for each trial included in their review and concluded that the TROPIC trial had a high risk of selection and performance/detection bias.

However, in Table 2, the authors made several assumptions about the methodology of the TROPIC trial, which we feel warrant clarification.

Perletti article assumption (Row 1): Risk of bias for the random sequence generation used in the TROPIC study was unclear. The dynamic allocation system was not a random sampling strategy in a strict sense and patients were not allocated randomly because allocation was determined on the basis of prognostic factors chosen as inclusion criteria.

Author correction: Random sequence generation for the TROPIC study was undertaken using stratified randomization by means of an Interactive Voice Response System (IVRS).

Stratification used two baseline factors (disease measurability and performance status) (1). A dynamic allocation was rarely instigated and was imposed only if there was an extreme assignment imbalance in a center.

Perletti article assumption (Row 2): Risk of bias for concealment of treatment allocation in TROPIC was high. Patients and treating physicians were not masked to treatment allocation.

Author correction: Although individual patients at individual sites were not blinded, a centralized IVRS was used in TROPIC, therefore patients and treating physicians were not aware of global enrollment information or the treatment allocation at other sites.

Perletti article assumption (Row 3): Risk of bias for the blinding of patients and personnel in TROPIC was high. The study had an open-label design. Although survival, biochemical findings and radiographic evidence could not have been influenced by the open-label setting, other endpoints (e.g., pain, quality of life) could have been prone to performance bias. Author correction: We agree that efficacy endpoints (overall survival [OS], prostate-specific antigen [PSA] levels, radiographic response/progression) were not impacted by the open-label study design or subject to bias. We note that quality of life data were not collected.

Perletti article assumption (Row 5): Risk of bias for any incomplete outcome data in the TROPIC trial was unclear because no detailed information about censoring rules was provided.

Author correction: This statement is puzzling. The primary endpoint of the TROPIC study was OS. The primary publication of TROPIC data states that OS data were censored at the last date the patient was known to be alive or at the analysis cut-off date, whichever was earliest (1). Almost all patients died before the end of the study or were censored at the study cut-off date. OS data were missing in very few patients.

Perletti article assumption (Row 7): Risk of bias for other factors, such as baseline imbalances, was unclear for TROPIC. In a long-term survival analysis, it was shown that $18 \%$ and $9 \%$ of patients in the cabazitaxel and mitoxantrone treatment arms, respectively, were alive at 24 months (2). At baseline, the presence of visceral metastases, which can be prognostic for OS, was higher in the mitoxantrone arm (20\%) compared with the cabazitaxel arm (15\%).

Author correction: Data for baseline visceral metastases in TROPIC are reported incorrectly by Perletti et al. Among patients receiving cabazitaxel $(n=378)$ and mitoxantrone $(n=377), 25 \%$ of patients in each treatment arm had baseline visceral metastases ( $\mathrm{n}=94$ in each arm) (1), thus no imbalance exists. After 24 months, almost twice as many patients were alive in the cabazitaxel arm $(n=60,15.9 \%)$ compared with the mitoxantrone arm $(n=31,8.2 \%)$, demonstrating a long-term OS benefit for cabazitaxel versus mitoxantrone (2). In addition, a multivariate analysis supports the OS benefit of cabazitaxel (2).

(Remark 1)

Perletti et al. also assessed the effects of the interventions in the selected trials on OS and radiographic progression-free 
survival (rPFS), which was defined as the time between the date of randomization and the first date of radiographic progression. Perletti et al. stated that rPFS data were not available for the TROPIC study. In fact a composite PFS endpoint was reported for the TROPIC trial, defined as the time between randomization and progression measured by PSA progression, tumor/radiological progression, pain/symptomatic progression, or death (1).

Median time to tumor progression was also reported using RECIST criteria $(1,3)$. Thus, the definition of time to tumor progression used in the TROPIC study is aligned with the definition of rPFS used by Perletti et al., and these TROPIC data could have been included in their assessment.

(Remark 2)

Finally, in their conclusions, Perletti et al. note that compared with control cohorts in the different studies, survival advantages were 4.8 months for enzalutamide, 4.6 months for abiraterone and 2.4 months for cabazitaxel. We would like to point out that absolute differences in median OS between such different clinical trials should not be compared; furthermore it must be made clear that the control arms in these trials are quite different. The control arms of the enzalutamide and abiraterone clinical trials received placebo, whereas in the cabazitaxel trial the control arm received the cytotoxic agent, mitoxantrone, which has antitumor activity in some patients.

(Remark 3)

Overall, we commend Perletti et al. for their attempts to provide a detailed interpretation and comparison of key trials in the rapidly evolving field of $\mathrm{mCRPC}$ treatment. We hope that our clarifications about the design and conduct of the TROPIC study will enable its important findings to be better understood.

\section{REFERENCES}

1. de Bono JS, Oudard S, Ozguroglu M, et al. Prednisone plus cabazitaxel or mitoxantrone for metastatic castration-resistant prostate cancer progressing after docetaxel treatment: a randomized open-label trial. Lancet. 2010; 376:1147-54.

2. Bahl A, Oudard S, Tombal B, et al. Impact of cabazitaxel on 2-year survival and palliation of tumour-related pain in men with metastatic castration-resistant prostate cancer treated in the TROPIC trial. Ann Oncol. 2013; 24:2402-8.

3. Therasse P, Arbuck SG, Eisenhauer EA, et al. New guidelines to evaluate the response to treatment in solid tumors. European Organization for Research and Treatment of Cancer, National Cancer Institute of the United States, National Cancer Institute of Canada. J Natl Cancer Inst. 2000; 92:205-16.

\section{ACKNOWLEDGEMENTS}

The TROPIC study was sponsored by Sanofi. Editorial support in the preparation of this letter was provided by Danielle Lindley of MediTech Media funded by Sanofi. The authors, individuallyand collectively, are responsible for all content and editorial decisions and received no payment from Sanofi related to the development of this letter.

\section{Johann de Bono ${ }^{1}$, Liji Shen ${ }^{2}$, Oliver Sartor ${ }^{3}$ \\ ${ }^{1}$ Royal Marsden NHS Foundation Trust and The Institute of Cancer Research, Sutton, UK; ${ }^{2}$ Sanofi, Bridgewater, NJ, USA; ${ }^{3}$ Tulane University Medical School, New Orleans, LA, USA.}

\section{Correspondence}

Johann Sebastian de Bono, MBChB MSc PhD FRCP FMedSci (Corresponding Author) johann.de-bono@icr.ac.uk

Royal Marsden and The Institute of Cancer Research, London, Downs Road, Sutton, Surrey SM2 5PT, UK

Liji Shen

Liji.Shen@sanofi.com

Sanofi, Bridgewater, NJ, USA

Oliver Sartor

osartor@tulane.edu

Tulane University Medical School, New Orleans, LA, USA 